\title{
Duality Theory for Interval Linear Programming Problems
}

\author{
${ }^{1}$ G. Ramesh and ${ }^{2} \mathrm{~K}$. Ganesan \\ ${ }^{1,2}$ Department of Mathematics, Faculty of Engineering and Technology, SRM University, Kattankulathur, \\ Chennai - 603203, India.
}

\begin{abstract}
We define the primal and dual linear programming problems involving interval numbers as the way of traditional linear programming problems. We discuss the solution concepts of primal and dual linear programming problems involving interval numbers without converting them to classical linear programming problems. By introducing new arithmetic operations between interval numbers, we prove the weak and strong duality theorems. Complementary slackness theorem is also proved. A numerical example is provided to illustrate the theory developed in this paper.
\end{abstract}

Keywords: Interval Numbers, Interval Arithmetic, Linear Programming, Weak Duality, Strong Duality, Complementary Slackness.

\section{INTRODUCTION}

Linear programming is a most widely and successfully used decision tool in the quantitative analysis of practical problems where rational decisions have to be made. In order to solve a Linear Programming Problem, the decision parameters of the model must be fixed at crisp values. But to model real-life problems and perform computations we must deal with uncertainty and inexactness. These uncertainty and inexactness are due to measurement inaccuracy, simplification of physical models, variations of the parameters of the system, computational errors etc. Interval analysis is an efficient and reliable tool that allows us to handle such problems effectively.

Linear programming problems with interval coefficients have been studied by several authors, such as Atanu Sengupta et al. [2, 3], Bitran [5], Chanas and Kuchta [6], Nakahara et al. [20], Steuer [26] and Tong Shaocheng [31]. Numerous methods for comparison of interval numbers can be found as in Atanu Sengupta and Tapan Kumar Pal [2, 3], Ganesan and Veeramani [8, 9] etc.

By taking maximum value range and minimum value range inequalities as constraint conditions, Tong Shaocheng [31] reduced the interval linear programming problem in to two classical linear programming problems and obtained an optimal interval solution to it. Ramesh and Ganesan [24] proposed a method for solving interval number linear programming problems without converting them to classical linear programming problems.

The duality theory for inexact linear programming problems was proposed by Soyster [27-29] and Thuente [30]. Falk [7] provided some properties on this problem. However, Pomerol [23] pointed out some drawbacks of Soyster's results and provided some mild conditions to improve them. Masahiro Inuiguchia [14] et al has studied the duality of interval number linear programming problems through fuzzy linear programming problems. Bector and Chandra [4] introduced a pair of linear primal-dual problems under fuzzy environment and established the duality relationship between them. Hsien-Chung $\mathrm{Wu}[12,13]$ introduced the concept of scalar product for closed intervals in the objective and inequality constraints of the primal and dual linear programming problems with interval numbers. He introduced a solution concept that is essentially similar to the notion of nondominated solution in multiobjective programming problems by imposing a partial ordering on the set of all closed intervals. He then proved the weak and strong duality theorems for linear programming problems with interval numbers. Rohn [25] also discussed the duality in a interval linear programming problem with real-valued objective function. In this paper, we attempt to develop the duality theory for interval linear programming problems without converting them to classical linear programming problems.

The rest of this paper is organised as follows: In section 2, we recall the definitions of interval number linear programming, interval numbers and some related results of interval arithmetic on them. In section 3 , we define the interval number primal and dual linear programming problems as the way of traditional linear programming problems. We then prove the weak and strong duality theorems. Complementary Slackness theorem is also proved. In section 4, a numerical example is provided to illustrate the theory developed in this paper.

\section{PRELIMINARIES}

The aim of this section is to present some notations, notions and results which are of useful in our further consideration. 
Let $\operatorname{IR}=\left\{\tilde{a}=\left[a_{1}, a_{2}\right]: a_{1} \leq a_{2}\right.$ and $\left.a_{1}, a_{2} \in R\right\} \quad$ be the set of all proper intervals and $\overline{\mathrm{IR}}=\left\{\tilde{a}=\left[a_{1}, a_{2}\right]: a_{1}>a_{2}\right.$ and $\left.a_{1}, a_{2} \in R\right\}$ be the set of all improper intervals on the real line R. If $\tilde{a}=a_{1}=a_{2}=a$, then $\tilde{a}=[a, a]=a$ is a real number (or a degenerate interval). We shall use the terms "interval" and "interval number" interchangeably. The mid-point and width (or half-width) of an interval number are defined as

The mid-point and width (or half-width) of an interval number $\tilde{a}=\left[a_{1}, a_{2}\right]$ are defined as $m(\tilde{a})=\left(\frac{a_{1}+a_{2}}{2}\right)$ and $\mathrm{w}(\tilde{\mathrm{a}})=\left(\frac{\mathrm{a}_{2}-\mathrm{a}_{1}}{2}\right)$. The interval number $\tilde{\mathrm{a}}$ can also be expressed in terms of its midpoint and width as $\tilde{\mathrm{a}}=\left[\mathrm{a}_{1}, \mathrm{a}_{2}\right]=\langle\mathrm{m}(\tilde{\mathrm{a}}), \mathrm{w}(\tilde{\mathrm{a}})\rangle$.

\subsection{A New Interval Arithmetic}

Ming Ma et al.[18] have proposed a new fuzzy arithmetic based upon both location index and fuzziness index function. The location index number is taken in the ordinary arithmetic, whereas the fuzziness index functions are considered to follow the lattice rule which are the least upper bound and greatest lower bound in the lattice $L$. That is for $a, b \in L$ we define $a \vee b=\max \{a, b\}$ and $a \wedge b=\min \{a, b\}$.

For any two intervals $\tilde{\mathrm{a}}=\left[\mathrm{a}_{1}, \mathrm{a}_{2}\right], \tilde{\mathrm{b}}=\left[\mathrm{b}_{1}, \mathrm{~b}_{2}\right] \in \mathbb{I R}$ and for $* \in\{+,-, \cdot, \div\}$, the arithmetic operations on $\tilde{a}$ and $\tilde{b}$ are defined as:

$\tilde{a} * \tilde{b}=\left[a_{1}, a_{2}\right] *\left[b_{1}, b_{2}\right]=\langle m(\tilde{a}), w(\tilde{a})\rangle *\langle m(\tilde{b}), w(\tilde{b})\rangle=\langle m(\tilde{a}) * m(\tilde{b}), \max \{w(\tilde{a}), w(\tilde{b})\}\rangle$.

In particular

(i). Addition : $\tilde{a}+\tilde{b}=\langle m(\tilde{a}), w(\tilde{a})\rangle+\langle m(\tilde{b}), w(\tilde{b})\rangle=\langle m(\tilde{a})+m(\tilde{b}), \max \{w(\tilde{a}), w(\tilde{b})\}\rangle$.

(ii). Subtraction : $\tilde{a}-\tilde{b}=\langle m(\tilde{a}), w(\tilde{a})\rangle-\langle m(\tilde{b}), w(\tilde{b})\rangle=\langle m(\tilde{a})-m(\tilde{b}), \max \{w(\tilde{a}), w(\tilde{b})\}\rangle$.

(iv). Multiplication : $\tilde{a} \times \tilde{b}=\langle\mathrm{m}(\tilde{\mathrm{a}}), \mathrm{w}(\tilde{\mathrm{a}})\rangle \times\langle\mathrm{m}(\tilde{\mathrm{b}}), \mathrm{w}(\tilde{\mathrm{b}})\rangle=\langle\mathrm{m}(\tilde{\mathrm{a}}) \times \mathrm{m}(\tilde{\mathrm{b}}), \max \{\mathrm{w}(\tilde{\mathrm{a}}), \mathrm{w}(\tilde{\mathrm{b}})\}\rangle$.

(v). Division : $\tilde{\mathrm{a}} \div \tilde{\mathrm{b}}=\langle\mathrm{m}(\tilde{\mathrm{a}}), \mathrm{w}(\tilde{\mathrm{a}})\rangle \div\langle\mathrm{m}(\tilde{\mathrm{b}}), \mathrm{w}(\tilde{\mathrm{b}})\rangle=\langle\mathrm{m}(\tilde{\mathrm{a}}) \div \mathrm{m}(\tilde{\mathrm{b}}), \max \{\mathrm{w}(\tilde{\mathrm{a}}), \mathrm{w}(\tilde{\mathrm{b}})\}\rangle, \operatorname{provided} \mathrm{m}(\tilde{\mathrm{b}}) \approx \tilde{0}$.

\section{Main Results}

Now we are in a position to prove interval analogue of some important relationships between the primal and dual linear programming problems. We consider the primal and dual linear programming problems involving interval numbers as follows:

Consider the following linear programming problem involving interval numbers

(P)

$$
\max \tilde{\mathrm{z}} \approx \sum_{\mathrm{j}=1}^{\mathrm{n}} \tilde{\mathrm{c}}_{\mathrm{j}} \tilde{\mathrm{x}}_{\mathrm{j}}
$$

subject to $\sum_{\mathrm{j}=1}^{\mathrm{n}} \tilde{\mathrm{a}}_{\mathrm{ij}} \tilde{\mathrm{x}}_{\mathrm{j}} \preceq \tilde{\mathrm{b}}_{\mathrm{i}}, \mathrm{i}=1,2,3 \ldots \mathrm{m}$

and $\tilde{x}_{j} \succeq \tilde{0}$ for all $j=1,2,3, \ldots \ldots ., n$,

where $\tilde{a}_{i j}, \tilde{c}_{j}, \tilde{x}_{j}, \tilde{b}_{i} \in I R, i=1,2,3, \ldots \ldots . . m$ and $j=1,2,3, \ldots \ldots ., n$. rewritten as

We call the above problem $(\mathrm{P})$ as the primal interval linear programming problem, and it can be

(P) $\quad \max \tilde{z} \approx \tilde{\mathbf{c}} \tilde{\mathbf{x}} \quad$ subject to $\tilde{\mathrm{A}} \tilde{\mathbf{x}} \preceq \tilde{\mathbf{b}}$ and $\tilde{\mathbf{x}} \succeq \tilde{\mathbf{0}}$,

where $\tilde{\mathrm{A}}, \tilde{\mathbf{b}}, \tilde{\mathbf{c}}, \tilde{\mathbf{x}}$ are $(\mathrm{m} \times \mathrm{n}),(\mathrm{m} \times 1),(1 \times \mathrm{n}),(\mathrm{n} \times 1)$ matrices involving interval numbers.

$\mathrm{X}=\left\{\tilde{\mathbf{x}}=\left(\tilde{\mathrm{X}}_{1}, \tilde{\mathrm{X}}_{2}, \tilde{\mathrm{X}}_{3}, \cdots \ldots, \tilde{\mathbf{x}}_{\mathrm{n}}\right): \tilde{\mathrm{A}} \tilde{\mathbf{x}} \preceq \tilde{\mathbf{b}}, \tilde{\mathbf{x}} \succeq \tilde{\mathbf{0}}\right\}$ be the feasible region of problem (3.1). We say that $\tilde{\mathbf{x}}^{*}$ is a feasible solution to the primal problem (3.1), if $\tilde{\mathbf{x}}^{*} \in X$. A feasible solution $\tilde{\mathbf{x}}^{*} \in X$ is said to be

an optimum solution to the primal problem (3.1), $\tilde{\mathbf{c}} \tilde{\mathbf{x}}^{*} \succeq \tilde{\mathbf{c}} \tilde{\mathbf{x}}$ for all $\tilde{\mathbf{x}} \in \mathrm{X}$, where $\tilde{\mathbf{c}} \tilde{\mathbf{x}}=\sum_{\mathrm{j}=1}^{\mathrm{n}} \tilde{\mathrm{c}}_{\mathrm{j}} \tilde{\mathrm{x}}_{\mathrm{j}}=\left(\tilde{\mathrm{c}}_{1} \tilde{\mathrm{x}}_{1}+\tilde{\mathrm{c}}_{2} \tilde{\mathrm{x}}_{2}+\tilde{\mathrm{c}}_{3} \tilde{\mathrm{x}}_{3}+\ldots \ldots+\tilde{\mathrm{c}}_{\mathrm{n}} \tilde{\mathrm{x}}_{\mathrm{n}}\right)$ and $\tilde{\mathbf{c}}=\left(\tilde{\mathrm{c}}_{1}, \tilde{\mathrm{c}}_{2}, \tilde{\mathrm{c}}_{3}, \ldots \ldots ., \tilde{\mathrm{c}}_{\mathrm{n}}\right)$.

Now we consider the following linear programming problem involving interval numbers: 
(D) $\quad \min \tilde{\mathrm{w}} \approx \sum_{\mathrm{i}=1}^{\mathrm{m}} \tilde{\mathrm{b}}_{\mathrm{i}} \tilde{\mathrm{y}}_{\mathrm{i}}$

subject to $\sum_{i=1}^{m} \tilde{a}_{i j} \tilde{y}_{i} \succeq \tilde{c}_{j}, j=1,2,3 \ldots n$

and $\tilde{y}_{i} \succeq \tilde{0}$ for all $\mathrm{i}=1,2,3, \ldots \ldots, \mathrm{m}$,

where $\tilde{a}_{i j}, \tilde{c}_{j}, \tilde{x}_{j}, \tilde{b}_{i} \in I R, i=1,2,3, \ldots \ldots . . m$ and $j=1,2,3, \ldots \ldots ., n$.

We call the above problem (D) or (3.3) as the dual interval linear programming problem of the primal problem $(\mathrm{P})$, and it can be rewritten as

$$
\min \tilde{\mathrm{w}} \approx \tilde{\mathbf{b}} \tilde{\mathbf{y}} \quad \text { subject to } \tilde{\mathrm{A}} \tilde{\mathbf{y}} \succeq \tilde{\mathbf{c}} \text { and } \tilde{\mathbf{y}} \succeq \tilde{\mathbf{0}},
$$

where $\tilde{\mathrm{A}}, \tilde{\mathbf{b}}, \tilde{\mathbf{c}}, \tilde{\mathbf{y}}$ are $(\mathrm{m} \times \mathrm{n}),(1 \times \mathrm{m}),(\mathrm{n} \times 1),(\mathrm{m} \times 1)$ matrices involving interval numbers.

Let $\mathrm{Y}=\left\{\tilde{\mathbf{y}}=\left(\tilde{\mathrm{y}}_{1}, \tilde{\mathbf{y}}_{2}, \tilde{\mathbf{y}}_{3}, \cdots, \mathrm{y}_{\mathrm{m}}\right): \tilde{\mathrm{A}} \tilde{\mathbf{y}} \succeq \tilde{\mathbf{c}}, \tilde{\mathbf{y}} \succeq \tilde{\mathbf{0}}\right\}$ be the feasible region of problem (3.3). We say that $\tilde{\mathbf{y}}^{*}$ is a feasible solution to the dual problem (3.3), if $\tilde{\mathbf{y}}^{*} \in \mathrm{Y}$. A feasible solution $\tilde{\mathbf{y}}^{*} \in \mathrm{Y}$ is said to be an optimum solution to the dual (3.3) if $\tilde{\mathbf{b}} \tilde{\mathbf{y}}^{*} \preceq \tilde{\mathbf{b}} \tilde{\mathbf{y}}$ for all $\tilde{\mathbf{y}} \in \mathrm{Y}$, where $\tilde{\mathbf{b}} \tilde{\mathbf{y}}=\sum_{\mathrm{i}=1}^{\mathrm{m}} \tilde{\mathrm{b}}_{\mathrm{i}} \tilde{\mathrm{y}}_{\mathrm{i}}=\tilde{\mathrm{b}}_{1} \tilde{\mathrm{y}}_{1}+\tilde{\mathrm{b}}_{2} \tilde{\mathbf{y}}_{2}+\tilde{\mathrm{b}}_{3} \tilde{\mathbf{y}}_{3}+\cdots+\tilde{\mathrm{b}}_{\mathrm{m}} \mathrm{y}_{\mathrm{m}}$ and

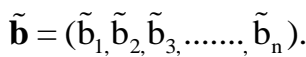

\section{Standard Form:}

For the general study, we convert the given interval number linear programming problem into its standard form as $\max \tilde{\mathbf{Z}} \approx \tilde{\mathbf{c}} \tilde{\mathbf{x}} \quad$ subject to $\tilde{\mathrm{A}} \tilde{\mathbf{x}} \approx \tilde{\mathbf{b}}$ and $\tilde{\mathbf{x}} \succeq \tilde{\mathbf{0}}$, where $\tilde{\mathrm{A}}, \tilde{\mathbf{b}}, \tilde{\mathbf{c}}, \tilde{\mathbf{x}}$ are $(\mathrm{m} \times \mathrm{n}),(\mathrm{m} \times 1),(1 \times \mathrm{n}),(\mathrm{n} \times 1)$ matrices consisting of interval numbers.

Definition 3.1. Let $\tilde{\mathbf{x}}=\left(\tilde{\mathrm{x}}_{1}, \tilde{\mathrm{x}}_{2}, \tilde{\mathrm{x}}_{3}, \ldots, \tilde{\mathrm{x}}_{\mathrm{n}}\right)$ solves $\tilde{\mathrm{A}} \tilde{\mathbf{x}} \approx \tilde{\mathbf{b}}$. If all $\tilde{\mathrm{x}}_{\mathrm{j}} \approx\left[-\alpha_{\mathrm{j}}, \alpha_{\mathrm{j}}\right]$ for some $\alpha_{\mathrm{j}} \geq 0$, then $\tilde{\mathbf{x}}$ is said to be a basic solution. If $\tilde{\mathrm{x}}_{\mathrm{j}} \approx\left[-\alpha_{\mathrm{j}}, \alpha_{\mathrm{j}}\right]$ for some $\alpha_{\mathrm{j}} \geq 0$, then $\tilde{\mathbf{x}}$ has some non-zero components, say $\tilde{\mathbf{x}}_{1}, \tilde{\mathbf{x}}_{2}, \tilde{\mathbf{x}}_{3,} \ldots . ., \tilde{\mathbf{x}}_{\mathrm{k}}, 1 \leq \mathrm{k} \leq \mathrm{n}$. Then $\tilde{\mathrm{A}} \tilde{\mathbf{x}} \approx \tilde{\mathbf{b}}$ can be written as:

$$
\tilde{\mathbf{a}}_{1} \tilde{\mathbf{x}}_{1}+\tilde{\mathbf{a}}_{2} \tilde{\mathbf{x}}_{2}+\tilde{\mathbf{a}}_{\mathbf{3}} \tilde{\mathbf{x}}_{3}+\ldots+\tilde{\mathbf{a}}_{\mathbf{k}} \tilde{\mathbf{x}}_{\mathrm{k}}+\tilde{\mathbf{a}}_{\mathbf{k}+\mathbf{1}}\left[-\beta_{\mathrm{k}+1}, \beta_{\mathrm{k}+1}\right]+\tilde{\mathbf{a}}_{\mathbf{k}+2}\left[-\beta_{\mathrm{k}+2}, \beta_{\mathrm{k}+2}\right]+\ldots+\tilde{\mathbf{a}}_{\mathbf{n}}\left[-\beta_{\mathrm{n}}, \beta_{\mathrm{n}}\right] \approx \tilde{\mathbf{b}}
$$

If the columns $\tilde{\mathbf{a}}_{\mathbf{1}}, \tilde{\mathbf{a}}_{\mathbf{2}}, \tilde{\mathbf{a}}_{\mathbf{3}}, \ldots, \tilde{\mathbf{a}}_{\mathbf{k}}$ corresponding to these non-zero components $\tilde{\mathbf{x}}_{1}, \tilde{\mathbf{x}}_{2}, \tilde{\mathbf{x}}_{3}, \ldots . ., \tilde{\mathbf{x}}_{\mathrm{k}}$ are linearly independent, then $\tilde{\mathbf{x}}$ is said to be a basic solution.

Remark 3.1. Given a system of $m$ simultaneous linear equations involving interval numbers in $n$ unknowns $(\mathrm{m} \leq \mathrm{n}) \quad \tilde{\mathrm{A}} \tilde{\mathbf{x}} \approx \tilde{\mathbf{b}}, \tilde{\mathbf{b}} \in \mathrm{IR}^{\mathrm{m}}$, where $\tilde{\mathrm{A}}$ is an $(\mathrm{m} \times \mathrm{n})$ interval matrix and rank of $\tilde{\mathrm{A}}$ is $\mathrm{m}$. Let $\tilde{\mathrm{B}}$ be any $(\mathrm{m} \times \mathrm{m})$ interval matrix formed by $m$ linearly independent columns of $\tilde{\mathrm{A}}$.

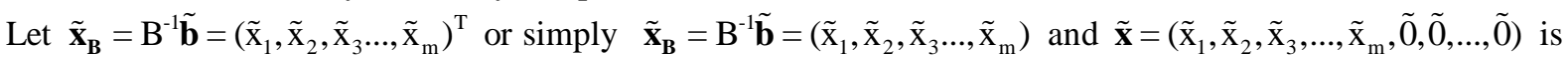
a basic solution. In this case, we also say that $\tilde{\mathbf{x}}_{\mathbf{B}}$ is a basic solution.

Theorem 3.1. Consider $\tilde{A} \tilde{\mathbf{x}} \approx \tilde{\mathbf{b}}$. where $\tilde{A}=\left(\tilde{a}_{\mathrm{ij}}\right)_{\mathrm{m} \times \mathrm{n}}, \tilde{\mathrm{a}}_{\mathrm{ij}} \in \operatorname{IR}$. Then $\tilde{\mathbf{x}}_{\mathbf{B}}=\mathrm{B}^{-1} \tilde{\mathbf{b}}$ is a solution of $\tilde{\mathrm{A}} \tilde{\mathbf{x}} \approx \tilde{\mathbf{b}}$.

Theorem 3. 2 (Weak duality theorem) If $\tilde{\mathbf{x}}=\left(\tilde{\mathrm{x}}_{1}, \tilde{\mathrm{x}}_{2}, \tilde{\mathrm{x}}_{3}, \ldots, \tilde{\mathrm{x}}_{\mathrm{n}}\right)$ is any feasible solution to the primal interval linear programming problem (3.1) and $\tilde{\mathbf{y}}=\left(\tilde{y}_{1}, \tilde{\mathbf{y}}_{2}, \tilde{\mathrm{y}}_{3}, \cdots, \tilde{\mathrm{y}}_{\mathrm{m}}\right)$ is any feasible solution to the dual interval linear programming problem (3.3), then $\tilde{\mathbf{c}} \tilde{\mathbf{x}} \preceq \tilde{\mathbf{b}} \tilde{\mathbf{y}}$ or $\sum_{\mathrm{j}=1}^{\mathrm{n}} \tilde{\mathbf{c}}_{\mathrm{j}} \tilde{\mathbf{x}}_{\mathrm{j}} \preceq \sum_{\mathrm{i}=1}^{\mathrm{m}} \tilde{\mathrm{b}}_{\mathrm{i}} \tilde{\mathrm{y}}_{\mathrm{i}}$.

Proof. Since $\tilde{\mathbf{x}}$ is a feasible solution to the primal interval linear programming problem (3.1), we have $\sum_{\mathrm{j}=\mathrm{i}}^{\mathrm{n}} \tilde{\mathrm{a}}_{\mathrm{ij}} \tilde{\mathrm{x}}_{\mathrm{j}} \preceq \tilde{\mathrm{b}}_{\mathrm{i}}, \tilde{\mathrm{x}}_{\mathrm{j}} \succeq 0, \mathrm{i}=1,2,3, \cdots, \mathrm{m}$. $\sum_{\mathrm{j}=\mathrm{i}}^{\mathrm{n}}\left\langle\mathrm{m}\left(\tilde{\mathrm{a}}_{\mathrm{ij}}\right), \mathrm{w}\left(\tilde{\mathrm{a}}_{\mathrm{ij}}\right)\right\rangle\left\langle\mathrm{m}\left(\tilde{\mathrm{x}}_{\mathrm{j}}\right), \mathrm{w}\left(\tilde{\mathrm{x}}_{\mathrm{j}}\right)\right\rangle \preceq\left\langle\mathrm{m}\left(\tilde{\mathrm{b}}_{\mathrm{i}}\right), \mathrm{w}\left(\tilde{\mathrm{b}}_{\mathrm{i}}\right)\right\rangle, \tilde{\mathrm{x}}_{\mathrm{j}} \succeq 0, \mathrm{i}=1,2,3, \cdots, \mathrm{m}$.

Multiplying the $\mathrm{i}^{\text {th }}(\mathrm{i}=1,2,3, \cdots, \mathrm{m})$ primal constraint by $\tilde{\mathrm{y}}_{\mathrm{i}}=\left\langle\mathrm{m}\left(\tilde{\mathrm{y}}_{\mathrm{i}}\right), \mathrm{w}\left(\tilde{\mathrm{y}}_{\mathrm{i}}\right)\right\rangle$ and adding we have $\sum_{j=i}^{n}\left\langle\mathrm{~m}\left(\tilde{\mathrm{a}}_{\mathrm{ij}}\right), \mathrm{w}\left(\tilde{\mathrm{a}}_{\mathrm{ij}}\right)\right\rangle\left\langle\mathrm{m}\left(\tilde{\mathrm{x}}_{\mathrm{j}}\right), \mathrm{w}\left(\tilde{\mathrm{x}}_{\mathrm{j}}\right)\right\rangle\left\langle\mathrm{m}\left(\tilde{\mathrm{y}}_{\mathrm{i}}\right), \mathrm{w}\left(\tilde{y}_{\mathrm{i}}\right)\right\rangle \preceq\left\langle\mathrm{m}\left(\tilde{\mathrm{b}}_{\mathrm{i}}\right), \mathrm{w}\left(\tilde{\mathrm{b}}_{\mathrm{i}}\right)\right\rangle\left\langle\mathrm{m}\left(\tilde{\mathrm{y}}_{\mathrm{i}}\right), \mathrm{w}\left(\tilde{y}_{\mathrm{i}}\right)\right\rangle, \mathrm{i}=1,2,3, \cdots, \mathrm{m}$ $\sum_{\mathrm{i}=1}^{\mathrm{m}} \sum_{\mathrm{j}=\mathrm{i}}^{\mathrm{n}}\left\langle\mathrm{m}\left(\tilde{\mathrm{a}}_{\mathrm{ij}}\right), \mathrm{w}\left(\tilde{\mathrm{a}}_{\mathrm{ij}}\right)\right\rangle\left\langle\mathrm{m}\left(\tilde{\mathrm{x}}_{\mathrm{j}}\right), \mathrm{w}\left(\tilde{\mathrm{x}}_{\mathrm{j}}\right)\right\rangle\left\langle\mathrm{m}\left(\tilde{y}_{\mathrm{i}}\right), \mathrm{w}\left(\tilde{\mathrm{y}}_{\mathrm{i}}\right)\right\rangle \preceq \sum_{\mathrm{i}=1}^{\mathrm{m}}\left\langle\mathrm{m}\left(\tilde{\mathrm{b}}_{\mathrm{i}}\right), \mathrm{w}\left(\tilde{\mathrm{b}}_{\mathrm{i}}\right)\right\rangle\left\langle\mathrm{m}\left(\tilde{\mathrm{y}}_{\mathrm{i}}\right), \mathrm{w}\left(\tilde{\mathrm{y}}_{\mathrm{i}}\right)\right\rangle$

$$
\sum_{\mathrm{i}=1}^{\mathrm{m}} \sum_{\mathrm{j}=\mathrm{i}}^{\mathrm{n}}\left\langle\mathrm{m}\left(\tilde{\mathrm{a}}_{\mathrm{ij}}\right), \mathrm{w}\left(\tilde{\mathrm{a}}_{\mathrm{ij}}\right)\right\rangle\left\langle\mathrm{m}\left(\tilde{\mathrm{x}}_{\mathrm{j}}\right), \mathrm{w}\left(\tilde{\mathrm{x}}_{\mathrm{j}}\right)\right\rangle\left\langle\mathrm{m}\left(\tilde{\mathrm{y}}_{\mathrm{i}}\right), \mathrm{w}\left(\tilde{\mathrm{y}}_{\mathrm{i}}\right)\right\rangle \preceq \sum_{\mathrm{i}=1}^{\mathrm{m}} \tilde{\mathrm{b}}_{\mathrm{i}} \tilde{\mathrm{y}}_{\mathrm{i}}=\tilde{\mathbf{b}} \tilde{\mathbf{y}}
$$


Multiplying the $\mathrm{j}^{\text {th }}(\mathrm{j}=1,2,3, \ldots, \mathrm{n})$ dual constraint by $\tilde{\mathrm{x}}_{\mathrm{j}}=\left\langle\mathrm{m}\left(\tilde{\mathrm{x}}_{\mathrm{j}}\right), \mathrm{w}\left(\tilde{\mathrm{x}}_{\mathrm{j}}\right)\right\rangle$ and adding we have

$$
\begin{aligned}
& \sum_{i=1}^{m} \tilde{a}_{i j} \tilde{y}_{i} \tilde{x}_{j} \succeq \tilde{c}_{j} \tilde{x}_{j}, j=1,2,3, \ldots, n \\
& \sum_{i=1}^{m}\left\langle m\left(\tilde{a}_{i j}\right), w\left(\tilde{a}_{i j}\right)\right\rangle\left\langle m\left(\tilde{y}_{i}\right), w\left(\tilde{y}_{i}\right)\right\rangle\left\langle m\left(\tilde{x}_{j}\right), w\left(\tilde{x}_{j}\right)\right\rangle \succeq\left\langle m\left(\tilde{c}_{j}\right), w\left(\tilde{c}_{j}\right)\right\rangle\left\langle m\left(\tilde{x}_{j}\right), w\left(\tilde{x}_{j}\right)\right\rangle, j=1,2,3, \ldots, n \\
& \sum_{j=1}^{n} \sum_{i=1}^{m}\left\langle m\left(\tilde{a}_{i j}\right), w\left(\tilde{a}_{i j}\right)\right\rangle\left\langle m\left(\tilde{y}_{i}\right), w\left(\tilde{y}_{i}\right)\right\rangle\left\langle m\left(\tilde{x}_{j}\right), w\left(\tilde{x}_{j}\right)\right\rangle \succeq \sum_{j=1}^{n}\left\langle m\left(\tilde{c}_{j}\right), w\left(\tilde{c}_{j}\right)\right\rangle\left\langle m\left(\tilde{x}_{j}\right), w\left(\tilde{x}_{j}\right)\right\rangle \\
& \sum_{i=1}^{m} \sum_{j=1}^{n}\left\langle m\left(\tilde{a}_{i j}\right), w\left(\tilde{a}_{i j}\right)\right\rangle\left\langle m\left(\tilde{y}_{i}\right), w\left(\tilde{y}_{i}\right)\right\rangle\left\langle m\left(\tilde{x}_{j}\right), w\left(\tilde{x}_{j}\right)\right\rangle \succeq \sum_{j=1}^{n}\left\langle m\left(\tilde{c}_{j}\right), w\left(\tilde{c}_{j}\right)\right\rangle\left\langle m\left(\tilde{x}_{j}\right), w\left(\tilde{x}_{j}\right)\right\rangle \\
& \sum_{i=1}^{m} \sum_{j=1}^{n}\left\langle m\left(\tilde{a}_{i j}\right), w\left(\tilde{a}_{i j}\right)\right\rangle\left\langle m\left(\tilde{y}_{i}\right), w\left(\tilde{y}_{i}\right)\right\rangle\left\langle m\left(\tilde{x}_{j}\right), w\left(\tilde{x}_{j}\right)\right\rangle \succeq \sum_{j=1}^{n} \tilde{c}_{j} \tilde{x}_{j}=\tilde{\mathbf{c}} \tilde{\mathbf{x}} .
\end{aligned}
$$

From equations (3.5) and (3.6), we have

$$
\begin{aligned}
& \sum_{\mathrm{j}=1}^{\mathrm{n}}\left\langle\mathrm{m}\left(\tilde{\mathrm{c}}_{\mathrm{j}}\right), \mathrm{w}\left(\tilde{\mathrm{c}}_{\mathrm{j}}\right)\right\rangle\left\langle\mathrm{m}\left(\tilde{\mathrm{x}}_{\mathrm{j}}\right), \mathrm{w}\left(\tilde{\mathrm{x}}_{\mathrm{j}}\right)\right\rangle \preceq \sum_{\mathrm{i}=1}^{\mathrm{m}} \sum_{\mathrm{j}=1}^{\mathrm{n}}\left\langle\mathrm{m}\left(\tilde{\mathrm{a}}_{\mathrm{ij}}\right), \mathrm{w}\left(\tilde{\mathrm{a}}_{\mathrm{ij}}\right)\right\rangle\left\langle\mathrm{m}\left(\tilde{\mathrm{y}}_{\mathrm{i}}\right), \mathrm{w}\left(\tilde{\mathrm{y}}_{\mathrm{i}}\right)\right\rangle\left\langle\mathrm{m}\left(\tilde{\mathrm{x}}_{\mathrm{j}}\right), \mathrm{w}\left(\tilde{\mathrm{x}}_{\mathrm{j}}\right)\right\rangle \\
& \preceq \sum_{\mathrm{i}=1}^{\mathrm{m}}\left\langle\mathrm{m}\left(\tilde{\mathrm{b}}_{\mathrm{i}}\right), \mathrm{w}\left(\tilde{\mathrm{b}}_{\mathrm{i}}\right)\right\rangle\left\langle\mathrm{m}\left(\tilde{\mathrm{y}}_{\mathrm{i}}\right), \mathrm{w}\left(\tilde{\mathrm{y}}_{\mathrm{i}}\right)\right\rangle \\
& \Rightarrow \sum_{\mathrm{j}=1}^{\mathrm{n}}\left\langle\mathrm{m}\left(\tilde{\mathrm{c}}_{\mathrm{j}}\right), \mathrm{w}\left(\tilde{\mathrm{c}}_{\mathrm{j}}\right)\right\rangle\left\langle\mathrm{m}\left(\tilde{\mathrm{x}}_{\mathrm{j}}\right), \mathrm{w}\left(\tilde{\mathrm{x}}_{\mathrm{j}}\right)\right\rangle \preceq \sum_{\mathrm{i}=1}^{\mathrm{m}}\left\langle\mathrm{m}\left(\tilde{\mathrm{b}}_{\mathrm{i}}\right), \mathrm{w}\left(\tilde{\mathrm{b}}_{\mathrm{i}}\right)\right\rangle\left\langle\mathrm{m}\left(\tilde{\mathrm{y}}_{\mathrm{i}}\right), \mathrm{w}\left(\tilde{\mathrm{y}}_{\mathrm{i}}\right)\right\rangle \\
& \tilde{\mathbf{c}} \tilde{\mathbf{x}}=\sum_{\mathrm{j}=1}^{\mathrm{n}} \tilde{\mathbf{c}}_{\mathrm{j}} \tilde{\mathbf{x}}_{\mathrm{j}} \preceq \sum_{\mathrm{i}=1}^{\mathrm{m}} \tilde{\mathrm{b}}_{\mathrm{i}} \tilde{\mathrm{y}}_{\mathrm{i}}=\tilde{\mathbf{b}} \tilde{\mathbf{y}} \Rightarrow \tilde{\mathbf{c}} \tilde{\mathbf{x}} \preceq \tilde{\mathbf{b}} \tilde{\mathbf{y}} .
\end{aligned}
$$

Proposition 3.1. Suppose that $\left.\tilde{\mathbf{x}}^{*}=\left(\tilde{\mathrm{x}}_{1}, \tilde{\mathrm{x}}_{2}, \tilde{\mathrm{x}}_{3}, \ldots, \tilde{\mathrm{x}}_{\mathrm{n}}\right)\right)$ and $\tilde{\mathbf{y}}^{*}=\left(\tilde{\mathrm{y}}_{1}, \tilde{\mathrm{y}}_{2}, \tilde{\mathrm{y}}_{3}, \cdots, \tilde{\mathrm{y}}_{\mathrm{m}}\right)$ are feasible solutions to the primal (3.1) and the dual (3.3) respectively, such that $\tilde{\mathbf{c}} \tilde{\mathbf{x}}^{*} \approx \tilde{\mathbf{b}} \tilde{\mathbf{y}}^{*}$, then $\tilde{\mathbf{x}}^{*}$ and $\tilde{\mathbf{y}}^{*}$ are optimal solutions to the primal and dual problems respectively.

Proof. Let $\tilde{\mathbf{c}} \tilde{\mathbf{x}}^{*} \approx \tilde{\mathbf{b}} \tilde{\mathbf{y}}^{*}$. From the weak duality theorem, we have $\tilde{\mathbf{c}} \tilde{\mathbf{x}}^{*} \approx \tilde{\mathbf{b}} \tilde{\mathbf{y}}^{*} \succeq \tilde{\mathbf{c}} \tilde{\mathbf{x}}$ for all $\tilde{\mathbf{x}} \in \mathrm{X} \Rightarrow \tilde{\mathbf{c}} \tilde{\mathbf{x}}^{*} \succeq \tilde{\mathbf{c}} \tilde{\mathbf{x}}$ for all $\tilde{\mathbf{x}} \in \mathrm{X}$. Similarly, $\tilde{\mathbf{b}} \tilde{\mathbf{y}}^{*} \approx \tilde{\mathbf{c}} \tilde{\mathbf{x}}^{*}$ for all $\tilde{\mathbf{y}}^{*}=\left(\tilde{\mathbf{y}}_{1}, \tilde{\mathbf{y}}_{2}, \tilde{\mathbf{y}}_{3}, \cdots, \tilde{\mathbf{y}}_{\mathrm{m}}\right) \in \mathrm{Y} \Rightarrow \tilde{\mathbf{b}} \tilde{\mathbf{y}}^{*} \preceq \tilde{\mathbf{b}} \tilde{\mathbf{y}}$ for all $\tilde{\mathbf{y}}^{*} \in \mathrm{Y}$.

Proposition 3.2. If $\tilde{\mathbf{c}} \tilde{\mathbf{x}} \approx \tilde{\mathbf{b}} \tilde{\mathbf{y}}$, then there exist $\tilde{\mathbf{x}}^{*} \in \mathrm{X}$ and $\tilde{\mathbf{y}}^{*} \in \mathrm{Y}$ such that $\tilde{\mathbf{c}} \tilde{\mathbf{x}}^{*} \succeq \tilde{\mathbf{c}} \tilde{\mathbf{x}}$ for all $\tilde{\mathbf{x}} \in \mathrm{X}$ and $\tilde{\mathbf{b}} \tilde{\mathbf{y}}^{*} \preceq \tilde{\mathbf{b}} \tilde{\mathbf{y}}$ for all $\tilde{\mathbf{y}}^{*} \in \mathrm{Y}$. That is $\tilde{\mathbf{x}}^{*}$ is an optimum solution to the primal problem (3.1) and $\tilde{\mathbf{y}}^{*}$ an optimum solution to the dual problem (3.3).

Proof. Since $\tilde{\mathbf{c}} \tilde{\mathbf{x}}-\tilde{\mathbf{b}} \tilde{\mathbf{y}} \approx \tilde{\mathbf{0}}$, there exist $\tilde{\mathbf{x}} \in \mathrm{X}$ and $\tilde{\mathbf{y}}^{*} \in \mathrm{Y}$ such that $\tilde{\mathbf{c}} \tilde{\mathbf{x}}^{*} \approx \tilde{\mathbf{b}} \tilde{\mathbf{y}}^{*}$. Then the results follow immediately from proposition (3.1).

\section{Theorem 3. 3 (Strong duality theorem)}

If $\tilde{\mathbf{x}}=\left(\tilde{x}_{1}, \tilde{x}_{2}, \tilde{x}_{3}, \ldots \tilde{x}_{n}\right)$ is an optimal solution to the primal problem (3.1), then there exit a feasible solution $\tilde{\mathbf{y}}=\left(\tilde{y}_{1}, \tilde{y}_{2}, \tilde{y}_{3}, \ldots \tilde{y}_{m}\right)$ to the dual problem (3.3) such that $\tilde{\mathbf{c}} \tilde{\mathbf{x}} \approx \tilde{\mathbf{b}} \tilde{\mathbf{y}}$.

Proof: We convert the primal problem (3.1) to its standard form by adding slack variables as follows:

$$
\max \tilde{\mathbf{z}} \approx \sum_{\mathrm{j}=1}^{\mathrm{n}} \tilde{\mathrm{c}}_{\mathrm{j}} \tilde{\mathrm{x}}_{\mathrm{j}} \text { subject to } \sum_{\mathrm{j}=1}^{\mathrm{n}} \tilde{\mathrm{a}}_{\mathrm{ij}} \tilde{\mathrm{x}}_{\mathrm{j}}+\tilde{\mathrm{x}}_{\mathrm{n}+\mathrm{i}} \approx \tilde{\mathrm{b}}_{\mathrm{i}}, \mathrm{i}=1,2, \ldots, \mathrm{m} \text { and } \quad \tilde{\mathrm{x}}_{\mathrm{j}} \succeq \tilde{0}
$$

for all $\mathrm{j}=1,2, \ldots, \mathrm{n}, \mathrm{n}+1, \ldots, \mathrm{n}+\mathrm{m}$, where $\tilde{\mathrm{x}}_{\mathrm{n}+\mathrm{i}}$ are slack variables.

That is $\max \tilde{\mathbf{z}} \approx \tilde{\mathbf{c}} \tilde{\mathbf{x}}$ subject to $\tilde{A} \tilde{\mathbf{x}} \approx \tilde{\mathbf{b}}$ and $\tilde{\mathbf{x}} \succeq \tilde{\mathbf{0}}$,

where $\tilde{A}, \tilde{\mathbf{b}}, \tilde{\mathbf{c}}, \tilde{\mathbf{x}}$ are $(\mathrm{m} \times(\mathrm{n}+\mathrm{m})),(\mathrm{m} \times 1),(1 \times(\mathrm{n}+\mathrm{m})),((\mathrm{n}+\mathrm{m}) \times 1)$ matrices consisting of interval numbers. Let $\tilde{\mathbf{x}}_{\mathrm{B}}=\tilde{\mathrm{B}}^{-1} \tilde{\mathbf{b}}$ is an optimal basic feasible solution to (3.8), where $\mathrm{B}$ is the corresponding basis matrix, $\quad \tilde{\mathbf{c}}_{\mathrm{B}}$ is the cost vector corresponding to $\tilde{\mathbf{x}}_{\mathrm{B}}$. We know that $\tilde{\mathrm{z}}_{\mathrm{j}}=\tilde{\mathbf{c}}_{\mathrm{B}} \tilde{\mathrm{y}}_{\mathrm{j}}$, so that $\tilde{\mathrm{y}}_{\mathrm{j}} \tilde{\mathrm{B}}=\tilde{\mathrm{B}}^{-1} \tilde{\mathbf{a}}_{\mathrm{j}}$. Also $\left(\tilde{z}_{j}-\tilde{c}_{j}\right)=\left(\tilde{c}_{B} \tilde{y}_{j}-\tilde{c}_{j}\right)=\left\{\begin{array}{l}\tilde{c}_{B} \tilde{B}^{-1} \tilde{a}_{j}, \text { for } j=1,2,3, \ldots, n . \\ \left(\tilde{c}_{B} \tilde{B}^{-1} \tilde{e}_{j}-\tilde{0}\right), \text { for } j=n+1, n+2, n+3, \ldots, n+m\end{array}\right.$

Since $\tilde{\mathbf{x}}_{\mathrm{B}}=\tilde{\mathrm{B}}^{-1} \tilde{\mathbf{b}}$ is an optimal basic feasible solution to (3.8), we have $\left(\tilde{\mathrm{z}}_{\mathrm{j}}-\tilde{\mathrm{c}}_{\mathrm{j}}\right) \succeq \tilde{0}$ for all $\mathrm{j}$. So that 


$$
\begin{aligned}
\left(\tilde{\mathbf{z}}_{\mathrm{j}}-\tilde{\mathbf{c}}_{\mathrm{j}}\right) \succeq \tilde{0} & \Rightarrow\left(\tilde{\mathbf{c}}_{\mathrm{B}} \tilde{\mathrm{B}}^{-1} \tilde{\mathbf{a}}_{\mathrm{j}}-\tilde{\mathbf{c}}_{\mathrm{j}}\right) \succeq \tilde{0} \text { and }\left(\tilde{\mathbf{c}}_{\mathrm{B}} \tilde{\mathrm{B}}^{-1} \tilde{\mathrm{e}}_{\mathrm{j}}-\tilde{0}\right) \succeq \tilde{0} \\
& \Rightarrow\left(\tilde{\mathbf{c}}_{\mathrm{B}} \tilde{\mathrm{B}}^{-1} \tilde{\mathbf{a}}_{\mathrm{j}}-\tilde{\mathbf{c}}_{\mathrm{j}}\right) \succeq \tilde{0} \text { and } \tilde{\mathbf{c}}_{\mathrm{B}} \tilde{\mathrm{B}}^{-1} \succeq \tilde{\mathbf{0}} \Rightarrow\left(\tilde{\mathbf{c}}_{\mathrm{B}} \tilde{\mathrm{B}}^{-1} \tilde{\mathrm{A}}-\tilde{\mathbf{c}}_{\mathrm{j}}\right) \succeq \tilde{0} \text { and } \tilde{\mathbf{c}}_{\mathrm{B}} \tilde{\mathrm{B}}^{-1} \succeq \tilde{\mathbf{0}} . \\
& \Rightarrow \tilde{\mathbf{c}}_{\mathrm{B}} \tilde{\mathrm{B}}^{-1} \tilde{\mathrm{A}} \succeq \tilde{\mathbf{c}} \text { and } \tilde{\mathbf{c}}_{\mathrm{B}} \tilde{\mathrm{B}}^{-1} \succeq \tilde{\mathbf{0}} .
\end{aligned}
$$

Suppose that $\tilde{\mathbf{y}}=\tilde{\mathbf{c}}_{\tilde{\mathrm{B}}} \tilde{\mathrm{B}}^{-1}$, then $\tilde{\mathbf{y}} \succeq \tilde{\mathbf{0}}$ and $\tilde{\mathbf{c}}_{\mathrm{B}} \tilde{\mathrm{B}}^{-1} \tilde{\mathrm{A}} \succeq \tilde{\mathbf{c}} \Rightarrow \tilde{\mathbf{y}} \tilde{\mathrm{A}} \succeq \tilde{\mathbf{c}} \Rightarrow \tilde{\mathrm{A}} \tilde{\mathbf{y}} \succeq \tilde{\mathbf{c}} \Rightarrow \tilde{\mathbf{y}}$ is a feasible solution to the dual. Also $\tilde{\mathbf{b}} \tilde{\mathbf{y}}=\tilde{\mathbf{y}} \tilde{\mathbf{b}}=\tilde{\mathbf{c}}_{\mathrm{B}} \tilde{\mathrm{B}}^{-1} \tilde{\mathbf{b}}=\tilde{\mathbf{c}}_{\mathrm{B}} \tilde{\mathrm{B}} \approx \tilde{\mathbf{c}} \tilde{\mathbf{x}}$, when ever $\tilde{\mathbf{x}}$ is an optimal solution to the primal (3.8). Hence $\tilde{\mathbf{c}} \tilde{\mathbf{x}} \approx \tilde{\mathbf{b}} \tilde{\mathbf{y}}$.

\section{Theorem 3.4. (Complementary Slackness theorem)}

If $\tilde{\mathbf{x}}^{*}=\left(\tilde{\mathrm{x}}_{1}, \tilde{\mathrm{x}}_{2}, \tilde{\mathrm{x}}_{3}, \ldots, \tilde{\mathrm{x}}_{\mathrm{n}}\right)$ is a feasible solution to the primal (3.1) and $\tilde{\mathbf{y}}^{*}=\left(\tilde{\mathrm{y}}_{1}, \tilde{\mathrm{y}}_{2}, \tilde{\mathrm{y}}_{3}, \cdots, \tilde{\mathrm{y}}_{\mathrm{m}}\right)$ is a feasible solution to the dual (3.3), then they must satisfy the so-called complementary slackness conditions:

(i). If $\tilde{y}_{\mathrm{i}}^{*} \succ \tilde{0}$, then $\sum_{\mathrm{j}=1}^{\mathrm{n}} \tilde{\mathrm{a}}_{\mathrm{ij}} \tilde{\mathrm{x}}_{\mathrm{j}}^{*} \approx \tilde{\mathrm{b}}_{\mathrm{i}}$. (ii). $\sum_{\mathrm{j}=1}^{\mathrm{n}} \tilde{\mathrm{a}}_{\mathrm{ij}} \tilde{\mathrm{x}}_{\mathrm{j}}^{*} \prec \tilde{\mathrm{b}}_{\mathrm{i}}$, then $\tilde{\mathrm{y}}_{\mathrm{i}}^{*} \approx \tilde{0}$.

(iii). If $\tilde{\mathrm{x}}_{\mathrm{j}}^{*} \succ \tilde{0}$, then $\sum_{\mathrm{i}=1}^{\mathrm{m}} \tilde{\mathrm{a}}_{\mathrm{ij}} \tilde{y}_{\mathrm{i}}^{*} \approx \tilde{\mathrm{c}}_{\mathrm{j}}$. (iv). If $\sum_{\mathrm{i}=1}^{\mathrm{m}} \tilde{\mathrm{a}}_{\mathrm{ij}} \tilde{\mathrm{y}}_{\mathrm{i}}^{*} \succ \tilde{\mathrm{c}}_{\mathrm{j}}$, then $\tilde{\mathrm{x}}_{\mathrm{j}}^{*} \approx \tilde{0}$.

Proof. If $\tilde{\mathbf{x}}^{*}$ is a feasible solution to the primal (3.1) and $\tilde{\mathbf{y}}^{*}$ is a feasible solution to the dual (3.3), then from the strong duality theorem $\tilde{\mathbf{x}}^{*}$ is an optimal solution to the primal (3.1) and $\tilde{\mathbf{y}}^{*}$ is an optimal solution to the dual (3.3) such that from equation (3.7), we have

$$
\begin{aligned}
\sum_{\mathrm{j}=1}^{\mathrm{n}} \tilde{\mathrm{c}}_{\mathrm{j}} \tilde{\mathrm{x}}_{\mathrm{j}}^{*} \approx \sum_{\mathrm{i}=1}^{\mathrm{m}} \sum_{\mathrm{j}=\mathrm{i}}^{\mathrm{n}} \tilde{\mathrm{a}}_{\mathrm{ij}} \tilde{\mathrm{x}}_{\mathrm{j}}^{*} \tilde{\mathrm{y}}_{\mathrm{i}}^{*} \approx \sum_{\mathrm{i}=1}^{\mathrm{m}} \tilde{\mathrm{b}}_{\mathrm{i}} \tilde{\mathrm{y}}_{\mathrm{i}}^{*} . & \\
\text { i. e. } \sum_{\mathrm{j}=1}^{\mathrm{n}}\left\langle\mathrm{m}\left(\tilde{\mathrm{c}}_{\mathrm{j}}\right), \mathrm{w}\left(\tilde{\mathrm{c}}_{\mathrm{j}}\right)\right\rangle\left\langle\mathrm{m}\left(\tilde{\mathrm{x}}_{\mathrm{j}}^{*}\right), \mathrm{w}\left(\tilde{\mathrm{x}}_{\mathrm{j}}^{*}\right)\right\rangle & \approx \sum_{\mathrm{i}=1}^{\mathrm{m}} \sum_{\mathrm{j}=\mathrm{i}}^{\mathrm{n}}\left\langle\mathrm{m}\left(\tilde{\mathrm{a}}_{\mathrm{ij}}\right), \mathrm{w}\left(\tilde{\mathrm{a}}_{\mathrm{ij}}\right)\right\rangle\left\langle\mathrm{m}\left(\tilde{\mathrm{x}}_{\mathrm{j}}^{*}\right), \mathrm{w}\left(\tilde{\mathrm{x}}_{\mathrm{j}}^{*}\right)\right\rangle\left\langle\mathrm{m}\left(\tilde{\mathrm{y}}_{\mathrm{i}}^{*}\right), \mathrm{w}\left(\tilde{\mathrm{y}}_{\mathrm{i}}^{*}\right)\right\rangle \\
& \approx \sum_{\mathrm{i}=1}^{\mathrm{m}}\left\langle\mathrm{m}\left(\tilde{\mathrm{b}}_{\mathrm{i}}\right), \mathrm{w}\left(\tilde{\mathrm{b}}_{\mathrm{i}}\right)\right\rangle\left\langle\mathrm{m}\left(\tilde{\mathrm{y}}_{\mathrm{i}}^{*}\right), \mathrm{w}\left(\tilde{\mathrm{y}}_{\mathrm{i}}^{*}\right)\right\rangle .
\end{aligned}
$$

from equation (3.10), we have

$$
\begin{aligned}
& \Rightarrow \sum_{\mathrm{i}=1}^{\mathrm{m}} \sum_{\mathrm{j}=\mathrm{i}}^{\mathrm{n}}\left\langle\mathrm{m}\left(\tilde{\mathrm{a}}_{\mathrm{ij}}\right), \mathrm{w}\left(\tilde{\mathrm{a}}_{\mathrm{ij}}\right)\right\rangle\left\langle\mathrm{m}\left(\tilde{\mathrm{x}}_{\mathrm{j}}^{*}\right), \mathrm{w}\left(\tilde{\mathrm{x}}_{\mathrm{j}}^{*}\right)\right\rangle\left\langle\mathrm{m}\left(\tilde{\mathrm{y}}_{\mathrm{i}}^{*}\right), \mathrm{w}\left(\tilde{\mathrm{y}}_{\mathrm{i}}^{*}\right)\right\rangle \approx \sum_{\mathrm{i}=1}^{\mathrm{m}}\left\langle\mathrm{m}\left(\tilde{\mathrm{b}}_{\mathrm{i}}\right), \mathrm{w}\left(\tilde{\mathrm{b}}_{\mathrm{i}}\right)\right\rangle\left\langle\mathrm{m}\left(\tilde{\mathrm{y}}_{\mathrm{i}}^{*}\right), \mathrm{w}\left(\tilde{\mathrm{y}}_{\mathrm{i}}^{*}\right)\right\rangle . \\
& \Rightarrow \sum_{\mathrm{i}=1}^{\mathrm{m}} \sum_{\mathrm{i}=\mathrm{i}}^{\mathrm{n}}\left\langle\mathrm{m}\left(\tilde{\mathrm{a}}_{\mathrm{ij}}\right), \mathrm{w}\left(\tilde{\mathrm{a}}_{\mathrm{ij}}\right)\right\rangle\left\langle\mathrm{m}\left(\tilde{\mathrm{x}}_{\mathrm{j}}^{*}\right), \mathrm{w}\left(\tilde{\mathrm{x}}_{\mathrm{j}}^{*}\right)\right\rangle\left\langle\mathrm{m}\left(\tilde{\mathrm{y}}_{\mathrm{i}}^{*}\right), \mathrm{w}\left(\tilde{\mathrm{y}}_{\mathrm{i}}^{*}\right)\right\rangle-\sum_{\mathrm{i}=1}^{\mathrm{m}}\left\langle\mathrm{m}\left(\tilde{\mathrm{b}}_{\mathrm{i}}\right), \mathrm{w}\left(\tilde{\mathrm{b}}_{\mathrm{i}}\right)\right\rangle\left\langle\mathrm{m}\left(\tilde{\mathrm{y}}_{\mathrm{i}}^{*}\right), \mathrm{w}\left(\tilde{\mathrm{y}}_{\mathrm{i}}^{*}\right)\right\rangle \approx 0 . \\
& \Rightarrow \sum_{\mathrm{i}=1}^{\mathrm{m}}\left[\sum_{\mathrm{j}=\mathrm{i}}^{\mathrm{n}}\left(\left\langle\mathrm{m}\left(\tilde{\mathrm{a}}_{\mathrm{ij}}\right), \mathrm{w}\left(\tilde{\mathrm{a}}_{\mathrm{ij}}\right)\right\rangle\left\langle\mathrm{m}\left(\tilde{\mathrm{x}}_{\mathrm{j}}^{*}\right), \mathrm{w}\left(\tilde{\mathrm{x}}_{\mathrm{j}}^{*}\right)\right\rangle\right)-\left\langle\mathrm{m}\left(\tilde{\mathrm{b}}_{\mathrm{i}}\right), \mathrm{w}\left(\tilde{\mathrm{b}}_{\mathrm{i}}\right)\right\rangle\right]\left\langle\mathrm{m}\left(\tilde{\mathrm{y}}_{\mathrm{i}}^{*}\right), \mathrm{w}\left(\tilde{\mathrm{y}}_{\mathrm{i}}^{*}\right)\right\rangle \approx 0 \text {. } \\
& \Rightarrow\left[\sum_{\mathrm{j}=\mathrm{i}}^{\mathrm{n}}\left(\left\langle\mathrm{m}\left(\tilde{\mathrm{a}}_{\mathrm{ij}}\right), \mathrm{w}\left(\tilde{\mathrm{a}}_{\mathrm{ij}}\right)\right\rangle\left\langle\mathrm{m}\left(\tilde{\mathrm{x}}_{\mathrm{j}}^{*}\right), \mathrm{w}\left(\tilde{\mathrm{x}}_{\mathrm{j}}^{*}\right)\right\rangle\right)-\left\langle\mathrm{m}\left(\tilde{\mathrm{b}}_{\mathrm{i}}\right), \mathrm{w}\left(\tilde{\mathrm{b}}_{\mathrm{i}}\right)\right\rangle\right]\left\langle\mathrm{m}\left(\tilde{\mathrm{y}}_{\mathrm{i}}^{*}\right), \mathrm{w}\left(\tilde{\mathrm{y}}_{\mathrm{i}}^{*}\right)\right\rangle \approx 0 . \\
& \Rightarrow \sum_{\mathrm{j}=\mathrm{i}}^{\mathrm{n}}\left(\left\langle\mathrm{m}\left(\tilde{\mathrm{a}}_{\mathrm{ij}}\right), \mathrm{w}\left(\tilde{\mathrm{a}}_{\mathrm{ij}}\right)\right\rangle\left\langle\mathrm{m}\left(\tilde{\mathrm{x}}_{\mathrm{j}}^{*}\right), \mathrm{w}\left(\tilde{\mathrm{x}}_{\mathrm{j}}^{*}\right)\right\rangle\right)-\left\langle\mathrm{m}\left(\tilde{\mathrm{b}}_{\mathrm{i}}\right), \mathrm{w}\left(\tilde{\mathrm{b}}_{\mathrm{i}}\right)\right\rangle \approx 0 \text { (or) }\left\langle\mathrm{m}\left(\tilde{\mathrm{y}}_{\mathrm{i}}^{*}\right), \mathrm{w}\left(\tilde{\mathrm{y}}_{\mathrm{i}}^{*}\right)\right\rangle \approx 0 \text {. } \\
& \Rightarrow \sum_{\mathrm{j}=\mathrm{i}}^{\mathrm{n}}\left\langle\mathrm{m}\left(\tilde{\mathrm{a}}_{\mathrm{ij}}\right), \mathrm{w}\left(\tilde{\mathrm{a}}_{\mathrm{ij}}\right)\right\rangle\left\langle\mathrm{m}\left(\tilde{\mathrm{x}}_{\mathrm{j}}^{*}\right), \mathrm{w}\left(\tilde{\mathrm{x}}_{\mathrm{j}}^{*}\right)\right\rangle \approx\left\langle\mathrm{m}\left(\tilde{\mathrm{b}}_{\mathrm{i}}\right), \mathrm{w}\left(\tilde{\mathrm{b}}_{\mathrm{i}}\right)\right\rangle \text { (or) }\left\langle\mathrm{m}\left(\tilde{\mathrm{y}}_{\mathrm{i}}^{*}\right), \mathrm{w}\left(\tilde{\mathrm{y}}_{\mathrm{i}}^{*}\right)\right\rangle \approx 0 \text {. } \\
& \Rightarrow \sum_{\mathrm{j}=\mathrm{i}}^{\mathrm{n}} \tilde{\mathrm{a}}_{\mathrm{ij}} \tilde{\mathrm{x}}_{\mathrm{j}}^{*} \approx \tilde{\mathrm{b}}_{\mathrm{i}} \quad \text { (or) } \tilde{\mathrm{y}}_{\mathrm{i}}^{*} \approx \tilde{0}
\end{aligned}
$$

So, (i) if $\tilde{\mathrm{y}}_{\mathrm{i}}^{*} \succ \tilde{0}$, then $\sum_{\mathrm{j}=\mathrm{i}}^{\mathrm{n}} \tilde{\mathrm{a}}_{\mathrm{ij}} \tilde{\mathrm{x}}_{\mathrm{j}}^{*} \approx \tilde{\mathrm{b}}_{\mathrm{i}}$ and (ii) if $\sum_{\mathrm{j}=\mathrm{i}}^{\mathrm{n}} \tilde{\mathrm{a}}_{\mathrm{ij}} \tilde{\mathrm{x}}_{\mathrm{j}}^{*} \prec \tilde{\mathrm{b}}_{\mathrm{i}}$, then $\tilde{\mathrm{y}}_{\mathrm{i}}^{*} \approx \tilde{0}$.

Similarly from equation (3.10), we have $\sum_{\mathrm{i}=1}^{\mathrm{m}} \sum_{\mathrm{j}=\mathrm{i}}^{\mathrm{n}} \tilde{\mathrm{a}}_{\mathrm{ij}} \tilde{\mathrm{x}}_{\mathrm{j}} \tilde{\mathrm{y}}_{\mathrm{i}}^{*} \approx \sum_{\mathrm{j}=1}^{\mathrm{n}} \tilde{\mathrm{c}}_{\mathrm{j}} \tilde{\mathrm{x}}_{\mathrm{j}}^{*}$

That is $\sum_{\mathrm{i}=1}^{\mathrm{m}} \sum_{\mathrm{j}=\mathrm{i}}^{\mathrm{n}}\left\langle\mathrm{m}\left(\tilde{\mathrm{a}}_{\mathrm{ij}}\right), \mathrm{w}\left(\tilde{\mathrm{a}}_{\mathrm{ij}}\right)\right\rangle\left\langle\mathrm{m}\left(\tilde{\mathrm{x}}_{\mathrm{j}}^{*}\right), \mathrm{w}\left(\tilde{\mathrm{x}}_{\mathrm{j}}^{*}\right)\right\rangle\left\langle\mathrm{m}\left(\tilde{\mathrm{y}}_{\mathrm{i}}^{*}\right), \mathrm{w}\left(\tilde{\mathrm{y}}_{\mathrm{i}}^{*}\right)\right\rangle \approx \sum_{\mathrm{j}=1}^{\mathrm{n}}\left\langle\mathrm{m}\left(\tilde{\mathrm{c}}_{\mathrm{j}}\right), \mathrm{w}\left(\tilde{\mathrm{c}}_{\mathrm{j}}\right)\right\rangle\left\langle\mathrm{m}\left(\tilde{\mathrm{x}}_{\mathrm{j}}^{*}\right), \mathrm{w}\left(\tilde{\mathrm{x}}_{\mathrm{j}}^{*}\right)\right\rangle$

$$
\begin{aligned}
& \Rightarrow \sum_{\mathrm{i}=1}^{\mathrm{m}} \sum_{\mathrm{j}=\mathrm{i}}^{\mathrm{n}}\left\langle\mathrm{m}\left(\tilde{\mathrm{a}}_{\mathrm{ij}}\right), \mathrm{w}\left(\tilde{\mathrm{a}}_{\mathrm{ij}}\right)\right\rangle\left\langle\mathrm{m}\left(\tilde{\mathrm{x}}_{\mathrm{j}}^{*}\right), \mathrm{w}\left(\tilde{\mathrm{x}}_{\mathrm{j}}^{*}\right)\right\rangle\left\langle\mathrm{m}\left(\tilde{\mathrm{y}}_{\mathrm{i}}^{*}\right), \mathrm{w}\left(\tilde{\tilde{\mathrm{y}}}_{\mathrm{i}}^{*}\right)\right\rangle-\sum_{\mathrm{j}=1}^{\mathrm{n}}\left\langle\mathrm{m}\left(\tilde{\mathrm{c}}_{\mathrm{j}}\right), \mathrm{w}\left(\tilde{\mathrm{c}}_{\mathrm{j}}\right)\right\rangle\left\langle\mathrm{m}\left(\tilde{\mathrm{x}}_{\mathrm{j}}^{*}\right), \mathrm{w}\left(\tilde{\mathrm{x}}_{\mathrm{j}}^{*}\right)\right\rangle \approx \tilde{0} \\
& \Rightarrow \sum_{\mathrm{j}=\mathrm{i}}^{\mathrm{n}}\left[\sum_{\mathrm{i}=1}^{\mathrm{m}} \tilde{\mathrm{a}}_{\mathrm{ij}} \tilde{\mathrm{y}}_{\mathrm{i}}^{*}-\tilde{\mathrm{c}}_{\mathrm{j}}\right] \tilde{\mathrm{x}}_{\mathrm{j}}^{*} \approx \tilde{0} \Rightarrow\left[\sum_{\mathrm{i}=1}^{\mathrm{m}} \tilde{\mathrm{a}}_{\mathrm{ij}} \tilde{\mathrm{y}}_{\mathrm{i}}^{*}-\tilde{\mathrm{c}}_{\mathrm{j}}\right] \tilde{\mathrm{x}}_{\mathrm{j}}^{*} \approx \tilde{0} \\
& \Rightarrow \sum_{\mathrm{i}=1}^{\mathrm{m}} \tilde{\mathrm{a}}_{\mathrm{ij}} \tilde{\mathrm{y}}_{\mathrm{i}}^{*}-\tilde{\mathrm{c}}_{\mathrm{j}} \approx \tilde{0} \text { (or) } \tilde{\mathrm{x}}_{\mathrm{j}}^{*} \approx \tilde{0} \Rightarrow \sum_{\mathrm{i}=1}^{\mathrm{m}} \tilde{\mathrm{a}}_{\mathrm{ij}} \tilde{\mathrm{y}}_{\mathrm{i}}^{*} \approx \tilde{\mathrm{c}}_{\mathrm{j}} \text { (or) } \tilde{\mathrm{x}}_{\mathrm{j}}^{*} \approx \tilde{0}
\end{aligned}
$$

So (iii). If $\tilde{\mathrm{x}}_{\mathrm{j}}^{*} \succ \tilde{0}$, then $\sum_{\mathrm{i}=1}^{\mathrm{m}} \tilde{\mathrm{a}}_{\mathrm{ij}} \tilde{\mathrm{y}}_{\mathrm{i}}^{*} \approx \tilde{\mathrm{c}}_{\mathrm{j}}$ and (iv). If $\sum_{\mathrm{i}=1}^{\mathrm{m}} \tilde{\mathrm{a}}_{\mathrm{ij}} \tilde{\mathrm{y}}_{\mathrm{i}}^{*} \succ \tilde{\mathrm{c}}_{\mathrm{j}}$, then $\tilde{\mathrm{x}}_{\mathrm{j}}^{*} \approx \tilde{0}$. 


\section{Numerical Examples}

Example 4.1. Consider the following interval number linear programming problem:

$$
\begin{aligned}
& \text { Max } \tilde{\mathrm{z}}=[29,31] \tilde{\mathrm{x}}_{1}+[22,24] \tilde{\mathrm{x}}_{2}+[28,30] \tilde{\mathrm{x}}_{3} \\
& \text { subject to constraints } 6 \tilde{\mathrm{x}}_{1}+5 \tilde{\mathrm{x}}_{2}+3 \tilde{\mathrm{x}}_{3} \preceq[25,27] \\
& \quad 4 \tilde{\mathrm{x}}_{1}+2 \tilde{\mathrm{x}}_{2}+5 \tilde{\mathrm{x}}_{3} \preceq[6,8] \\
& \text { and } \tilde{\mathrm{x}}_{1}, \tilde{\mathrm{x}}_{2}, \tilde{\mathrm{x}}_{3} \succeq 0 .
\end{aligned}
$$

We call

primal problem. Then the corresponding dual problem is given by

(D)

$$
\begin{gathered}
\text { Min } \tilde{\mathrm{w}} \approx[25,27] \tilde{\mathrm{y}}_{1}+[6,8] \tilde{\mathrm{y}}_{2} \\
\text { subject to constraints 6 } \tilde{\mathrm{y}}_{1}+4 \tilde{\mathrm{y}}_{2} \succeq[29,31] \\
5 \tilde{\mathrm{y}}_{1}+2 \tilde{\mathrm{y}}_{2} \succeq[22,24] \\
3 \tilde{\mathrm{y}}_{1}+5 \tilde{\mathrm{y}}_{2} \succeq[28,30] \\
\text { and } \tilde{\mathrm{y}}_{1}, \tilde{\mathrm{y}}_{2} \succeq \tilde{0} .
\end{gathered}
$$

\section{(i). Optimal solution to the primal interval number linear programming problem:}

Let us apply the interval version of simplex algorithm and the new interval arithmetic to solve the primal problem. The standard form of the given primal interval number linear programming problem based upon both location index (mid point) and fuzziness index function (width) as:

$$
\begin{aligned}
& \operatorname{Max} \tilde{\mathrm{z}} \approx\langle 30,1\rangle \tilde{\mathrm{x}}_{1}+\langle 23,1\rangle \tilde{\mathrm{x}}_{2}+\langle 29,1\rangle \tilde{\mathrm{x}}_{3}+0 \tilde{\mathrm{s}}_{1}+0 \tilde{\mathrm{s}}_{2} \\
& \text { subject to constraints } 6 \tilde{\mathrm{x}}_{1}+5 \tilde{\mathrm{x}}_{2}+3 \tilde{\mathrm{x}}_{3}+\tilde{\mathrm{s}}_{1}+0 \tilde{\mathrm{s}}_{2} \approx\langle 26,1\rangle \\
& \begin{array}{ll} 
& 4 \tilde{\mathrm{x}}_{1}+2 \tilde{\mathrm{x}}_{2}+5 \tilde{\mathrm{x}}_{4}+0 \tilde{\mathrm{s}}_{1} \\
\text { and } \quad & \tilde{\mathrm{x}}_{1}, \tilde{\mathrm{x}}_{2}, \tilde{\mathrm{x}}_{3}, \tilde{\mathrm{s}}_{1}, \tilde{\mathrm{s}}_{2} \succeq \tilde{0} .
\end{array}
\end{aligned}
$$

Initial iteration: Initial basic feasible solution is given by $\tilde{\mathrm{s}}_{1}=\langle 26,1\rangle, \tilde{\mathrm{s}}_{2}=\langle 7,1\rangle$.

$$
\begin{aligned}
& \begin{array}{ccccccccc} 
& & \tilde{\mathrm{c}}_{\mathrm{j}} & \langle 30,1\rangle & \langle 23,1\rangle & \langle 29,1\rangle & 0 & 0 & \\
\tilde{\mathrm{c}}_{\mathrm{B}} & \tilde{\mathrm{y}}_{\mathrm{B}} & \tilde{\mathrm{x}}_{\mathrm{B}} & \tilde{\mathrm{x}}_{1} & \tilde{\mathrm{x}}_{2} & \tilde{\mathrm{x}}_{3} & \tilde{\mathrm{s}}_{1} & \tilde{\mathrm{s}}_{2} & \theta \\
0 & \tilde{\mathrm{s}}_{1} & \langle 26,1\rangle & 6 & 5 & 3 & 1 & 0 & \langle 4.33,1\rangle \\
0 & \tilde{\mathrm{s}}_{2} & \langle 7,1\rangle & (4) & 2 & 5 & 0 & 1 & \langle 1.75,1\rangle
\end{array} \\
& \begin{array}{cccccc}
\tilde{\mathrm{z}}_{\mathrm{j}} & 0 & 0 & 0 & 0 & 0 \\
\left(\tilde{\mathrm{z}}_{\mathrm{j}}-\tilde{\mathrm{c}}_{\mathrm{j}}\right) & \langle-30,1\rangle & \langle-23,1\rangle & \langle-29,1\rangle & 0 & 0
\end{array}
\end{aligned}
$$

Since $\left(\tilde{z}_{j}-\tilde{c}_{j}\right) \prec \tilde{0}$, for some $j$, the current basic feasible solution is not Optimal.

First iteration: Here $\tilde{\mathrm{s}}_{2}$ leaves the basis and $\tilde{\mathrm{x}}_{1}$ enters in to the basis

$$
\begin{array}{ccccccccc} 
& & \tilde{\mathrm{c}}_{\mathrm{j}} & \langle 30,1\rangle & \langle 23,1\rangle & \langle 29,1\rangle & 0 & 0 & \\
\tilde{\mathrm{c}}_{\mathrm{B}} & \tilde{\mathrm{y}}_{\mathrm{B}} & \tilde{\mathrm{x}}_{\mathrm{B}} & \tilde{\mathrm{x}}_{1} & \tilde{\mathrm{x}}_{2} & \tilde{\mathrm{x}}_{3} & \tilde{\mathrm{s}}_{1} & \tilde{\mathrm{s}}_{2} & \theta \\
0 & \tilde{\mathrm{s}}_{1} & \langle 15.5,1\rangle & 0 & 2 & -4.5 & 1 & -1.5 & \langle 7.75,1\rangle \\
\langle 30,1\rangle & \tilde{\mathrm{x}}_{1} & \langle 1.75,1\rangle & 1 & 0.5 & 1.25 & 0 & 0.25 & \langle 3.5,1\rangle \\
& & \tilde{\mathrm{z}}_{\mathrm{j}} & \langle 30,1\rangle & \langle 15,1\rangle & \langle 37.5,1\rangle & \langle 0,0\rangle & \langle 7.5,1\rangle & \\
& & \left(\tilde{\mathrm{z}}_{\mathrm{j}}-\tilde{\mathrm{c}}_{\mathrm{j}}\right) & \langle 0,0\rangle & \langle-8,1\rangle & \langle 8.5,1\rangle & \langle 0,0\rangle & \langle 7.5,1\rangle &
\end{array}
$$

Since $\left(\tilde{\mathrm{z}}_{\mathrm{j}}-\tilde{\mathrm{c}}_{\mathrm{j}}\right) \prec \tilde{0}$, for some $\mathrm{j}$, the current basic feasible solution is not Optimal.

The improved basic feasible solution is given by $\tilde{\mathrm{s}}_{1}=\langle 15.5,1\rangle, \tilde{\mathrm{x}}_{1}=\langle 1.75,1\rangle$.

Second iteration: Here $\tilde{\mathrm{x}}_{1}$ leaves the basis and $\tilde{\mathrm{x}}_{2}$ enters in to the basis

$$
\begin{array}{ccccccccc} 
& & \tilde{\mathrm{c}}_{\mathrm{j}} & \langle 30,1\rangle & \langle 23,1\rangle & \langle 29,1\rangle & 0 & 0 & \\
\tilde{\mathrm{c}}_{\mathrm{B}} & \tilde{\mathrm{y}}_{\mathrm{B}} & \tilde{\mathrm{x}}_{\mathrm{B}} & \tilde{\mathrm{x}}_{1} & \tilde{\mathrm{x}}_{2} & \tilde{\mathrm{x}}_{3} & \tilde{\mathrm{s}}_{1} & \tilde{\mathrm{s}}_{2} & \theta \\
0 & \tilde{\mathrm{s}}_{1} & \langle 8.5,1\rangle & -4 & 0 & -9.5 & 1 & -2.5 & \\
\langle 23,1\rangle & \tilde{\mathrm{x}}_{2} & \langle 3.5,1\rangle & 2 & 1 & 2.5 & 0 & 0.5 & \\
& & \tilde{\mathrm{z}}_{\mathrm{j}} & \langle 46,1\rangle & \langle 23,1\rangle & \langle 57,1\rangle & \langle 0,0\rangle & \langle 11.5,1\rangle & \\
& & \left(\tilde{\mathrm{z}}_{\mathrm{j}}-\tilde{\mathrm{c}}_{\mathrm{j}}\right) & \langle 16,1\rangle & \langle 0,0\rangle & \langle 28.5,1\rangle & \langle 0,0\rangle & \langle 11.5,1\rangle &
\end{array}
$$


Since $\left(\tilde{z}_{j}-\tilde{c}_{j}\right) \succeq \tilde{0}$ for all $\mathrm{j}$, the current basic feasible solution is optimal. The optimal solution is $\tilde{s}_{1}=\langle 8.5,1\rangle, \tilde{x}_{2}=\langle 3.5,1\rangle$ and $\max \tilde{z}=\langle 80.5,1\rangle$.

Hence the optimal solution for the given primal interval number linear programming problem is $\tilde{\mathrm{x}}_{1}=[0,0], \tilde{\mathrm{x}}_{2}=[2.5,4.5]$ and $\max \tilde{\mathrm{z}}=[79.5,81.5]$.

(ii). Optimal solution to the dual interval number linear programming problem:

The standard form of the given dual interval number linear programming problem based upon both location index (mid point) and fuzziness index function (width) as:

(D)

$$
\begin{aligned}
& \min \tilde{\mathrm{w}} \approx\langle 26,1\rangle \tilde{\mathrm{y}}_{1}+\langle 7,1\rangle \tilde{\mathrm{y}}_{2}+0 \tilde{\mathrm{s}}_{1}+0 \tilde{\mathrm{s}}_{2}+0 \tilde{\mathrm{s}}_{3}+\mathrm{M} \tilde{\mathrm{R}}_{1}+\mathrm{MR} \tilde{\mathrm{R}}_{2}+\mathrm{M} \tilde{\mathrm{R}}_{3} \\
& \text { subject to } 6 \tilde{\mathrm{y}}_{1}+4 \tilde{\mathrm{y}}_{2}-\tilde{\mathrm{s}}_{1}+0 \tilde{\mathrm{s}}_{2}+0 \tilde{\mathrm{s}}_{3}+\mathrm{R}_{1}+0 \mathrm{R}_{2}+0 \mathrm{R}_{3} \approx\langle 30,1\rangle \\
& 5 \tilde{\mathrm{y}}_{1}+2 \tilde{\mathrm{y}}_{2}+0 \tilde{\mathrm{s}}_{1}-\tilde{\mathrm{s}}_{2}+0 \tilde{\mathrm{s}}_{3}+0 \mathrm{R}_{1}+\mathrm{R}_{2}+0 \mathrm{R}_{3} \approx\langle 23,1\rangle \\
& \quad 3 \tilde{\mathrm{y}}_{1}+5 \tilde{\mathrm{y}}_{2}+0 \tilde{\mathrm{s}}_{1}+0 \tilde{\mathrm{s}}_{2}-\tilde{\mathrm{s}}_{3}+0 \mathrm{R}_{1}+0 \mathrm{R}_{2}+\mathrm{R}_{3} \approx\langle 29,1\rangle \\
& \text { and } \tilde{\mathrm{y}}_{1}, \tilde{\mathrm{y}}_{2}, \tilde{\mathrm{s}}_{1}, \tilde{\mathrm{s}}_{2} \tilde{\mathrm{s}}_{3} \succeq 0
\end{aligned}
$$

\begin{tabular}{|c|c|c|c|c|c|c|c|c|c|c|c|}
\hline & & $\tilde{b}_{j}$ & $\langle 26,1\rangle$ & $\langle 7,1\rangle$ & 0 & 0 & 0 & M & M & M & \\
\hline$\tilde{\mathrm{C}}_{\mathrm{B}}$ & $\tilde{\mathrm{Y}}_{\mathrm{B}}$ & $\tilde{\mathrm{X}}_{\mathrm{B}}$ & $\tilde{\mathrm{y}}_{1}$ & $\tilde{y}_{2}$ & $\tilde{\mathrm{s}}_{1}$ & $\tilde{\mathrm{s}}_{2}$ & $\tilde{s}_{3}$ & $\tilde{\mathrm{R}}_{1}$ & $\tilde{\mathrm{R}}_{2}$ & $\tilde{\mathrm{R}}_{3}$ & $\theta$ \\
\hline $\mathrm{M}$ & $\tilde{\mathrm{R}}_{1}$ & $\langle 30,1\rangle$ & 6 & 4 & -1 & 0 & 0 & 1 & 0 & 0 & $\langle 5,1\rangle$ \\
\hline M & $\tilde{\mathrm{R}}_{2}$ & $\langle 23,1\rangle$ & (5) & 2 & 0 & -1 & 0 & 0 & 1 & 0 & $\langle 4.6,1\rangle$ \\
\hline M & $\tilde{\mathrm{R}}_{3}$ & $\langle 29,1\rangle$ & 3 & 5 & 0 & 0 & -1 & 0 & 0 & 1 & $\langle 9.6,1\rangle$ \\
\hline & & $\tilde{\mathrm{w}}_{\mathrm{j}}$ & $14 \mathrm{M}$ & $11 \mathrm{M}$ & $-\mathrm{M}$ & $-\mathrm{M}$ & $-\mathrm{M}$ & $\mathrm{M}$ & $\mathrm{M}$ & $\mathrm{M}$ & \\
\hline & & $\left(\tilde{w}_{j}-\tilde{b}_{j}\right)$ & $\langle 14 \mathrm{M}-26,1\rangle$ & $\langle 11 \mathrm{M}-7,1\rangle$ & $-\mathrm{M}$ & $-M$ & $-\mathrm{M}$ & 0 & 0 & 0 & \\
\hline
\end{tabular}

Initial iteration: Initial basic feasible solution is given by $\tilde{R}_{1}=\langle 30,1\rangle, \tilde{R}_{2}=\langle 23,1\rangle$ and $\tilde{R}_{3}=\langle 29,1\rangle$.

Since $\left(\tilde{\mathrm{w}}_{\mathrm{j}}-\tilde{\mathrm{b}}_{\mathrm{j}}\right) \succ \tilde{0}$, for some $\mathrm{j}$, the current basic feasible solution is not Optimal.

First iteration: Here $\tilde{\mathrm{R}}_{2}$ leaves the basis and $\tilde{\mathrm{y}}_{1}$ enters in to the basis

$$
\begin{array}{ccccccccccc} 
& & \tilde{\mathrm{b}}_{\mathrm{j}} & \langle 26,1\rangle & \langle 7,1\rangle & 0 & 0 & 0 & \mathrm{M} & \mathrm{M} & \\
\tilde{\mathrm{c}}_{\mathrm{B}} & \tilde{\mathrm{y}}_{\mathrm{B}} & \tilde{\mathrm{x}}_{\mathrm{B}} & \tilde{\mathrm{y}}_{1} & \tilde{\mathrm{y}}_{2} & \tilde{\mathrm{s}}_{1} & \tilde{\mathrm{s}}_{2} & \tilde{\mathrm{s}}_{3} & \tilde{\mathrm{R}}_{1} & \tilde{\mathrm{R}}_{2} & \theta \\
\mathrm{M} & \tilde{\mathrm{R}}_{1} & \langle 2.4,1\rangle & 0 & 1.0 & -1 & 1.2 & 0 & 1 & 0 & \langle 1.5,1\rangle \\
\langle 26,1\rangle & \tilde{\mathrm{y}}_{1} & \langle 4.6,1\rangle & 1 & 0.4 & 0 & 0 & 0 & 0 & 0 & \langle 11.5,1\rangle \\
\mathrm{M} & \tilde{\mathrm{R}}_{3} & \langle 15.2,1\rangle & 0 & 3.8 & 0 & 0 & -1 & 0 & 1 & \langle 4,1\rangle \\
& & \tilde{\mathrm{w}}_{\mathrm{j}} & \langle 26,1\rangle & \langle 5.4 \mathrm{M}+10.4,1\rangle & -\mathrm{M} & \langle 1.8 \mathrm{M}-5.2,1\rangle & -\mathrm{M} & \mathrm{M} & \mathrm{M} & \\
& & \left(\tilde{\mathrm{w}}_{\mathrm{j}}-\tilde{\mathrm{b}}_{\mathrm{j}}\right) & \langle 0,0\rangle & \langle 5.4+3.4,1\rangle & -\mathrm{M} & \langle 1.8 \mathrm{M}-5.2,1\rangle & -\mathrm{M} & 0 & 0 &
\end{array}
$$

Since $\left(\tilde{\mathrm{w}}_{\mathrm{j}}-\tilde{\mathrm{b}}_{\mathrm{j}}\right) \succ \tilde{0}$, for some $\mathrm{j}$, the current basic feasible solution is not Optimal.

The improved basic feasible solution is given by $\tilde{\mathrm{R}}_{1}=\langle 2.4,1\rangle, \tilde{\mathrm{y}}_{1}=\langle 4.6,1\rangle, \tilde{\mathrm{R}}_{3}=\langle 15.2,1\rangle$.

Second iteration: Here $\tilde{R}_{1}$ leaves the basis and $\tilde{y}_{2}$ enters in to the basis

$$
\begin{array}{cccccccccc} 
& & \tilde{\mathrm{b}}_{\mathrm{j}} & \langle 26,1\rangle & \langle 7,1\rangle & 0 & 0 & 0 & \mathrm{M} & \\
\tilde{\mathrm{c}}_{\mathrm{B}} & \tilde{\mathrm{y}}_{\mathrm{B}} & \tilde{\mathrm{x}}_{\mathrm{B}} & \tilde{\mathrm{y}}_{1} & \tilde{\mathrm{y}}_{2} & \tilde{\mathrm{s}}_{1} & \tilde{\mathrm{s}}_{2} & \tilde{\mathrm{s}}_{3} & \tilde{\mathrm{R}}_{3} & \theta \\
\langle 7,1\rangle & \tilde{\mathrm{y}}_{2} & \langle 1.5,1\rangle & 0 & 1 & -0.62 & 0.75 & 0 & 0 & \ldots . . \\
\langle 26,1\rangle & \tilde{\mathrm{y}}_{1} & \langle 4,1\rangle & 1 & 0 & 0.25 & -0.5 & 0 & 0 & \langle 16,1\rangle \\
\mathrm{M} & \tilde{\mathrm{R}}_{3} & \langle 9.5,1\rangle & 0 & 0 & 2.37 & -2.25 & -1 & 1 & \langle 4,1\rangle \\
& & \tilde{\mathrm{w}}_{\mathrm{j}} & \langle 26,1\rangle & \langle 7,1\rangle & \langle 2.37 \mathrm{M}+2.12,1\rangle & \langle-2.25 \mathrm{M}-7.75,1\rangle & -\mathrm{M} & \mathrm{M} & \\
& & \left(\tilde{\mathrm{w}}_{\mathrm{j}}-\tilde{\mathrm{b}}_{\mathrm{j}}\right) & \langle 0,0\rangle & \langle 0,0\rangle & \langle 2.37 \mathrm{M}+2.12,1\rangle & \langle-2.25 \mathrm{M}-7.75,1\rangle & -\mathrm{M} & 0 &
\end{array}
$$

Since $\left(\tilde{w}_{j}-\tilde{b}_{j}\right) \succ \tilde{0}$, for some $\mathrm{j}$, the current basic feasible solution is not Optimal. 
The improved basic feasible solution is given by $\tilde{\mathrm{y}}_{2}=\langle 1.5,1\rangle, \tilde{\mathrm{y}}_{1}=\langle 4,1\rangle, \tilde{\mathrm{R}}_{3}=\langle 9.5,1\rangle$.

Third iteration: Here $\tilde{\mathrm{R}}_{3}$ leaves the basis and $\tilde{\mathrm{s}}_{1}$ enters in to the basis

$\begin{array}{ccccccccc} & & \tilde{\mathrm{b}}_{\mathrm{j}} & \langle 26,1\rangle & \langle 7,1\rangle & 0 & 0 & 0 & \\ \tilde{\mathrm{c}}_{\mathrm{B}} & \tilde{\mathrm{y}}_{\mathrm{B}} & \tilde{\mathrm{x}}_{\mathrm{B}} & \tilde{\mathrm{y}}_{1} & \tilde{\mathrm{y}}_{2} & \tilde{\mathrm{s}}_{1} & \tilde{\mathrm{s}}_{2} & \tilde{\mathrm{s}}_{3} & \theta \\ \langle 7,1\rangle & \tilde{\mathrm{y}}_{2} & \langle 4,1\rangle & 0 & 1 & 0 & 0.16 & -0.26 & \ldots \\ \langle 26,1\rangle & \tilde{\mathrm{y}}_{1} & \langle 3,1\rangle & 1 & 0 & 0 & -0.27 & 0.10 & \langle 28.57,1\rangle \\ \langle 0,0\rangle & \tilde{\mathrm{s}}_{1} & \langle 4,1\rangle & 0 & 0 & 1 & -0.94 & -0.42 & \ldots \\ & & \tilde{\mathrm{w}}_{\mathrm{j}} & \langle 26,1\rangle & \langle 7,1\rangle & \langle 0,0\rangle & \langle-5.9,1\rangle & \langle 0.88,1\rangle & \\ & & \left(\tilde{\mathrm{w}}_{\mathrm{j}}-\tilde{\mathrm{b}}_{\mathrm{j}}\right) & \langle 0,0\rangle & \langle 0,0\rangle & \langle 0,0\rangle & \langle-5.9,1\rangle & \langle 0.88,1\rangle & \end{array}$

Since $\left(\tilde{w}_{j}-\tilde{b}_{j}\right) \succ \tilde{0}$, for some $\mathrm{j}$, the current basic feasible solution is not Optimal.

The improved basic feasible solution is given by $\tilde{\mathrm{y}}_{2}=\langle 4,1\rangle, \tilde{\mathrm{y}}_{1}=\langle 3,1\rangle, \tilde{\mathrm{s}}_{1}=\langle 4,1\rangle$.

Fourth iteration: Here $\tilde{y}_{1}$ leaves the basis and $\tilde{s}_{3}$ enters in to the basis

$\begin{array}{ccccccccc} & & \tilde{\mathrm{b}}_{\mathrm{j}} & \langle 26,1\rangle & \langle 7,1\rangle & 0 & 0 & 0 & \\ \tilde{\mathrm{c}}_{\mathrm{B}} & \tilde{\mathrm{y}}_{\mathrm{B}} & \tilde{\mathrm{x}}_{\mathrm{B}} & \tilde{\mathrm{y}}_{1} & \tilde{\mathrm{y}}_{2} & \tilde{\mathrm{s}}_{1} & \tilde{\mathrm{s}}_{2} & \tilde{\mathrm{s}}_{3} & \theta \\ \langle 7,1\rangle & \tilde{\mathrm{y}}_{2} & \langle 11.51,1\rangle & 2.50 & 1 & 0 & -0.5 & 0 & \ldots \\ \langle 0,0\rangle & \tilde{\mathrm{s}}_{3} & \langle 28.57,1\rangle & 9.52 & 0 & 0 & -2.57 & 1 & \ldots \\ \langle 0,0\rangle & \tilde{\mathrm{s}}_{1} & \langle 16.02,1\rangle & 4 & 0 & 1 & -2.02 & 0 & \ldots \\ & & \tilde{\mathrm{w}}_{\mathrm{j}} & \langle 17.5,1\rangle & \langle 7,1\rangle & \langle 0,0\rangle & \langle-3.5,1\rangle & \langle 0,0\rangle & \\ & & \left(\tilde{\mathrm{w}}_{\mathrm{j}}-\tilde{\mathrm{b}}_{\mathrm{j}}\right) & \langle-8.5,1\rangle & \langle 0,0\rangle & \langle 0,0\rangle & \langle-3.5,1\rangle & \langle 0,0\rangle & \end{array}$

Since $\left(\tilde{\mathrm{w}}_{\mathrm{j}}-\tilde{\mathrm{b}}_{\mathrm{j}}\right) \prec \tilde{0}$, for all $\mathrm{j}$, the current basic feasible solution is Optimal. The optimal solution is $\tilde{\mathrm{y}}_{2}=\langle 11.51,1\rangle, \tilde{\mathrm{s}}_{3}=\langle 28.57,1\rangle, \tilde{\mathrm{s}}_{1}=\langle 16.02,1\rangle$ and $\min \tilde{\mathrm{w}}=\langle 80.5,1\rangle$.

Hence the optimal solution for the dual interval number linear programming problem is $\tilde{y}_{1}=[0,0], \quad \tilde{y}_{2}=[10.5,12.5], \quad \tilde{y}_{3}=[0,0]$ and $\min \tilde{\mathrm{w}}=[79.5,81.5]$.

From the optimal solutions for the primal and dual interval number linear programming problem, we see that Primal : $\max \tilde{z}=[79.5,81.5]$ and Dual : $\min \tilde{w}=[795,81.5]$.

Hence Primal : $\max \tilde{z}=[79.5,81.5]=$ Dual: $\min \tilde{w}$

We see that both primal and dual problems have optimal solutions and the two optimal values are equal. Also both optimal solutions obey the strong duality theorem.

\section{CONCLUSION}

We introduced the notation of primal and dual linear programming problems involving interval numbers as the way of traditional linear programming problems. We discuss the solution concepts of primal and dual linear programming problems involving interval numbers without converting them to classical linear programming problems. Under new arithmetic operations between interval numbers, we have proved the weak and strong duality theorems. Complementary slackness theorem is also proved. These results will be useful for post optimality analysis. A numerical example is provided to show that both primal and dual problems have optimal solutions and the two optimal values are equal.

\section{Acknowledgements}

The authors are grateful to the anonymous referees and the editors for their constructive comments and suggestions.

\section{REFERENCES}

[1]. G. Alefeld and J. Herzberger, Introduction to Interval Computations, Academic Press, New York 1983.

[2]. Atanu Sengupta, Tapan Kumar Pal, Theory and Methodology: On comparing interval numbers, European Journal of Operational Research, 27 (2000), $28-43$.

[3]. Atanu Sengupta, Tapan Kumar Pal and Debjani Chakraborty, Interpretation of inequality constraints involving interval coefficients and a solution to interval linear programming, Fuzzy Sets and Systems, 119 (2001) 129-138.

[4]. Bector, C. R. and S. Chandra, "On Duality in Linear Programming under Fuzzy Environment," 
Fuzzy Sets and Systems 125 (2002), 317-325.

[5]. G. R. Bitran, Linear multiple objective problems with interval coefficients, Management Science, 26 (1980) 694 - 706.

[6]. S. Chanas and D. Kuchta, Multiobjective programming in optimization of interval objective functions - a generalized approach, European Journal of Operational Research, 94 (1996), 594-598.

[7]. Falk, J.E.: Exact solutions of inexact linear programs. Oper. Res. 24 (1976), 783-787.

[8]. K. Ganesan and P. Veeramani, On Arithmetic Operations of Interval Numbers, International Journal of Uncertainty, Fuzziness and Knowledge - Based Systems, 13 (6) (2005), 619 - 631.

[9]. K. Ganesan, On Some Properties of Interval Matrices, International Journal of Computational and Mathematical Sciences, 1 (2) (2007), 92 - 99.

[10]. E. Hansen, Global Optimization Using Interval Analysis, New York: Marcel Dekker, 1992

[11]. Hladik M., Optimal value range in interval linear programming, Faculty of Mathematics and Physics, Charles University, Prague, 2007.

[12]. Hsien-ChungWu, Duality theory for optimization problems with interval-valued objective functions. J. Optim.Theory Appl. 144 (2010), 615-628.

[13]. Hsien-Chung Wu, Duality Theory in Interval-Valued Linear Programming Problems, J Optim Theory Appl, 150 (2011), $298-316$.

[14]. Inuiguchi,M., J. Ramik, T. Tanino and M. Vlach, "Satisficing Solutions and Duality in Interval and Fuzzy Linear Programming", Fuzzy Sets and Systems 135 (2003), 151-177.

[15]. E. Kaucher, Interval analysis in extended interval space IR, Comput. Suppl. 2 (1980),33-49.

[16]. W. A. Lodwick and K. D. Jamison, Interval methods and fuzzy optimization, Int. J. Unccertainty, Fuzziness Knowledge-Based Systems, 5 (1997), 239-249.

[17]. R. E. Moore, Method and Application of Interval Analysis, SIAM Philadelphia, 1979.

[18]. Ming Ma, Menahem Friedman, Abraham kandel, A new fuzzy arithmetic, Fuzzy sets and systems, 108 (1999), 83-90

[19]. Mraz F., Calculating the exact bounds of optimal values in LP with interval coefficients, Annals of Operations Research, 81 (1998), 51 - 62.

[20]. Y. Nakahara, M. Sasaki and M. Gen, On the linear programming with interval coefficients, International Journal of Computers and Engineering, 23 (1992) 301-304.

[21]. T. Nirmala, D. Datta, H. S. Kushwaha and K. Ganesan, Inverse Interval Matrix: A New Approach, Applied Mathematical Sciences, 5 (13) (2011), 607 -624 .

[22]. Oliveira D. and Antunes C.H., Multiple objective linear programming models with interval coefficients - an illustrated overview, European Journal of Operational Research, 181(2007), 1434-1463.

[23]. Pomerol, J.C.: Constraint qualification for inexact linear programs. Oper. Res. 27 (1979), 843-847.

[24]. G. Ramesh and K. Ganesan, Interval Linear Programming with generalized interval arithmetic, international Journal of Scientific\& Engineering Research , 2 (11) (2011) ISSN 2229-5518.

[25]. Rohn, J, Duality in interval linear programming. In: Nickel, K.L.E. (ed.) Proceedings of an International Symposium on Interval Mathematics, pp. 521-529. Academic Press, New York (1980).

[26]. R.E. Steuer, Algorithm for linear programming problems with interval objective function coefficients, Mathematics of Operational Research, 6 (1981), $333-348$.

[27]. Soyster, A.L.: Convex programming with set-inclusive constraints and applications to inexact linear programming. Oper. Res. 21 (1973), 1154-1157.

[28]. Soyster, A.L.: A duality theory for convex programming with set-inclusive constraints. Oper. Res. 22 (1974), $892-898$ Erratum, $1279-1280$

[29]. Soyster, A.L.: Inexact linear programming with generalized resource sets. Eur. J. Oper. Res. 3 (1979), 316-321.

[30]. Thuente, D.J, Duality theory for generalized linear programs with computational methods. Oper. Res. 28 (1980), 1005-1011

[31]. Tong Shaocheng, Interval number and fuzzy number linear programming, Fuzzy Sets and Systems, 66 (1994) 301 - 306.

[32]. Thuente, D.J.: Duality theory for generalized linear programs with computational methods. Oper. Res. 28 (1980), 1005-1011.

[33]. M. Walk, Theory of Duahty in Mathematical Programmzng, Springer-Verlag, Wren, (1989). 